\title{
Modulation of Visual Responses by Gaze Direction in Human Visual Cortex
}

\author{
Elisha P. Merriam, ${ }^{1,2}$ Justin L. Gardner, ${ }^{3}$ J. Anthony Movshon, ${ }^{1}$ and David J. Heeger ${ }^{1,2}$ \\ ${ }^{1}$ Center for Neural Science, New York University, New York, New York 10003, ${ }^{2}$ Department of Psychology, New York University, New York, New York \\ 10003, and ${ }^{3}$ Gardner Research Unit, RIKEN Brain Science Institute, 2-1 Hirosawa, Wako, Saitama 351-0198, Japan
}

To locate visual objects, the brain combines information about retinal location and direction of gaze. Studies in monkeys have demonstrated that eye position modulates the gain of visual signals with "gain fields," so that single neurons represent both retinotopic location and eye position. We wished to know whether eye position and retinotopic stimulus location are both represented in human visual cortex. Using functional magnetic resonance imaging, we measured separately for each of several different gaze positions cortical responses to stimuli that varied periodically in retinal locus. Visually evoked responses were periodic following the periodic retinotopic stimulation. Only the response amplitudes depended on eye position; response phases were indistinguishable across eye positions. We used multivoxel pattern analysis to decode eye position from the spatial pattern of response amplitudes. The decoder reliably discriminated eye position in five of the early visual cortical areas by taking advantage of a spatially heterogeneous eye position-dependent modulation of cortical activity. We conclude that responses in retinotopically organized visual cortical areas are modulated by gain fields qualitatively similar to those previously observed neurophysiologically.

\section{Introduction}

How does the visual system represent stimulus location robust to changes in eye position? One possibility is that the brain combines retinotopic stimulus location with information about eye position to transform stimulus location from retinotopic coordinates to head-centered or body-centered coordinates. A coordinate system that is not yoked to the retina would be particularly useful for integrating visual information with input from other sensory systems or when planning limb movements to visual targets (Jay and Sparks, 1984; Soechting and Flanders, 1992; Stricanne et al., 1996; Groh et al., 2001).

An alternative possibility is that the brain represents stimulus location with gain fields (Andersen and Mountcastle, 1983). A neuron's receptive field captures how its responses depend on retinal stimulus location. Similarly, a neuron's gain field determines how its responses depend on eye position. For each stimulus location and each eye position, the neuron's response is the product of the receptive field and the gain field. The amplitude of a neuron's visually evoked responses are thus modulated by the static position of the eyes, so that retinal stimulus location, eye position, and head-centered stimulus location are simultaneously represented by a population of neurons with different receptive fields and different gain fields.

Gain fields were discovered in neurons in macaque parietal cortex (Andersen and Mountcastle, 1983) and have since been

\footnotetext{
Received Feb. 2, 2012; revised Feb. 28, 2013; accepted April 13, 2013.

Author contributions: E.P.M., J.L.G., J.A.M., and D.J.H. designed research; E.P.M. and J.L.G. performed research;

E.P.M., J.L.G., and D.J.H. analyzed data; E.P.M., J.L.G., J.A.M., and D.J.H. wrote the paper.

This work was supported by National Institutes of Health Grant R01-NS047493.

Correspondence should be addressed to Elisha P. Merriam, Center for Neural Science, New York University,

6 Washington Place, 8th Fl, New York, NY 10003. E-mail: eli@cns.nyu.edu.

DOI:10.1523/JNEUROSCI.0500-12.2013

Copyright $\odot 2013$ the authors $\quad 0270-6474 / 13 / 339879-11 \$ 15.00 / 0$
}

observed throughout the visual system (Galletti and Battaglini, 1989; Lal and Friedlander, 1990; Boussaoud et al., 1993; 1998; Galletti et al., 1995; Bremmer et al., 1998; Trotter and Celebrini, 1999; Rosenbluth and Allman, 2002; Sharma et al., 2003; Cassanello and Ferrera, 2007; Lehky et al., 2008). Functional magnetic resonance imaging (fMRI) studies in humans have demonstrated that changes in eye position affect activity in several cortical areas (Baker et al., 1999; DeSouza et al., 2002; Deutschländer et al., 2005; Bédard and Sanes, 2009; Williams and Smith, 2010; Bédard et al., 2011). However, it remains unclear from these studies whether the changes in fMRI activity reflected a true gain modulation of visually evoked activity. If so, then (1) the dependence on eye position should differ across the population of neurons, and (2) changes in eye position should result in a multiplicative change in the gain of visually evoked responses measured with fMRI.

Here, we report evidence for gain fields in human visual cortex. We measured cortical activity with fMRI while subjects viewed a visual stimulus that rotated periodically around a fixation point. The measurements were repeated with identical retinal stimulation while subjects held fixation at each of several eye positions. Response amplitudes depended on eye position, and eye positions could be decoded reliably from the spatial distribution of fMRI responses. This suggests that the eye-position dependence of cortical activity was spatially heterogeneous, consistent with gain fields that differed across the population of neurons. Response phases, on the other hand, were invariant to eye position and depended only on retinotopic stimulus location. This suggests that changes in eye position modulated the gain of visually evoked responses without shifting the receptive field locations. We conclude that each visual cortical area represents stimulus location in retinotopic coordinates, modulated by eye 
A

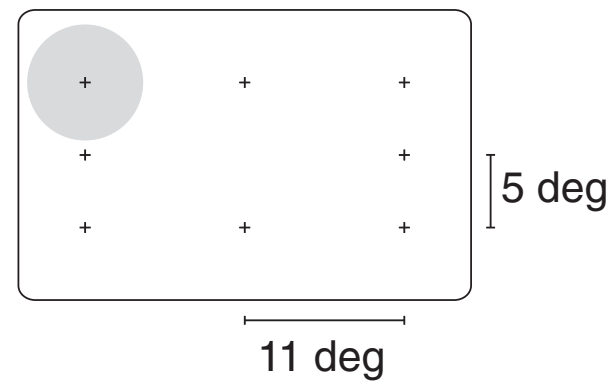

B

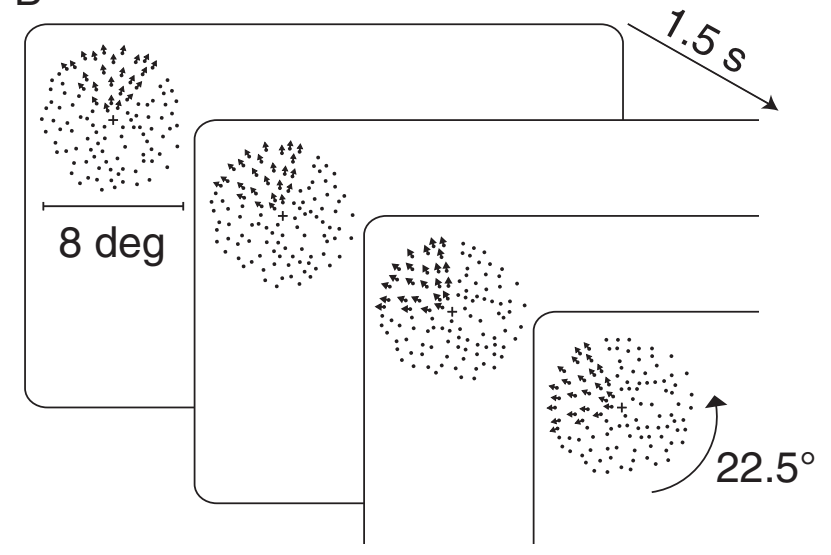

Figure 1. Schematic diagram of stimulus conditions. $\boldsymbol{A}$, In each run, subjects fixated a cross located at one of eight possible screen locations, indicated by crosses at $-11,0$, and $+11^{\circ}$ horizontal and $-5,0$, and $+5^{\circ}$ vertical, excluding the screen center. Gray shaded circle centered at the upper leftmost fixation position $\left(-11^{\circ},+5^{\circ}\right)$ indicates the relative size of the stimulus. $\boldsymbol{B}$, The stimulus consisted of a circular dot field ( $8^{\circ}$ diameter) in which one-quarter of the dot field (a $90^{\circ}$ wedge) moved radially, alternating between inward and outward motion every $750 \mathrm{~ms}$. The motion-defined wedge rotated $22.5^{\circ}$ every $1.5 \mathrm{~s}$, completing a full rotation in $24 \mathrm{~s}$. The wedge rotated through 10.5 cycles in a single scanning run. Eye position was held constant within each run and was varied across runs.

position, as expected if there are gain fields in human visual cortex.

\section{Materials and Methods}

Subjects. Five healthy male subjects aged 22-33 years participated in this study. All had normal or corrected-to-normal vision and provided prior written informed consent. Experimental procedures were in compliance with the safety guidelines for MRI research and were approved in advance by the University Committee on Activities Involving Human Subjects at New York University.

Stimulus and task. We measured visually driven cortical activity while systematically varying static orbital position. On each run, a cross appeared at one of eight possible screen locations, arranged in a $3 \times 3$ grid with horizontal eye positions of $-11 \mathrm{deg}, 0 \mathrm{deg}$, and $+11 \mathrm{deg}$, and vertical eye positions of $-5 \mathrm{deg}, 0 \mathrm{deg}$, and $+5 \mathrm{deg}$, excluding screen center (Fig. 1A). Subjects fixated the cross $(0.5 \mathrm{deg} \times 0.5 \mathrm{deg})$ for the duration of the run while viewing a visual stimulus. The stimulus consisted of a static dot field within an $8^{\circ}$-diameter circular aperture (Fig. $1 B$ ). Dots in onequarter of the field moved radially $(7 \% \mathrm{~s})$, alternating between inward and outward motion every $750 \mathrm{~ms}$, creating a motion-defined $90^{\circ}$ wedge. The wedge rotated $22.5^{\circ}$ about the fixation point every $1.5 \mathrm{~s}$, completing a full rotation every $24 \mathrm{~s}$. Each scanning run consisted of 10.5 rotations of the wedge stimulus and lasted for $4.2 \mathrm{~min}$. Eye position was held constant within a run. Eye position varied across runs according to a randomly shuffled order, with a different random order for each subject and scanning session. Subjects were instructed to fixate the cross for several seconds before each run to allow any hemodynamic response associated with the change in eye position to return to baseline. Each eye position was tested in two runs per scanning session and each subject participated in two sessions.

We used a motion-defined wedge in which the luminance and contrast were equal within the wedge and the rest of the stimulus aperture. This stimulus eliminated the possibility that a reflection of the stimulus (for example, off the scanner bore) or the visible edge of the screen could have yielded an artifact that would have confounded the interpretation of our results. If there had been any difference in contrast or luminance in the stimulus (as with conventional retinotopic mapping stimuli in which the wedge is high contrast against a uniform gray background), then any peripheral reflection of the stimulus would have modulated over time with the stimulus, and could have evoked activity in voxels corresponding to peripheral visual field locations. This peripheral stimulation would have constituted an additional source of cortical activity that could have varied with eye position, introducing a potential confound. By keeping contrast and luminance constant, the motion-defined wedge stimulus eliminated this potential source of extraneous stimulation, and ensured that changes in response amplitude were due entirely to differences in eye position, and not to differences in visual stimulation across eye positions.

Subjects performed a two-interval forced-choice luminance discrimination task at fixation, which was continuously adjusted to control task difficulty (Gardner et al., 2008). On every trial, the fixation cross was initially cyan $(500 \mathrm{~ms})$, then briefly dimmed during each of two target intervals $(100 \mathrm{~ms})$. The two target intervals were separated by a $500 \mathrm{~ms}$ period in which the cross was again cyan. After a final $500 \mathrm{~ms}$ cyan interval, the cross turned yellow to indicate the response interval. The subject was given $1 \mathrm{~s}$ to press one of two buttons indicating whether the cross was darker during its first or second dimming. If the subject responded correctly, the cross turned green for the remainder of the $1 \mathrm{~s}$ response period; otherwise, it turned red. The next trial began immediately so that the task was asynchronous with respect to the rotating wedge frequency and the MRI scanner repetition time. On each trial, the target luminance decrement was set by a two-down one-up staircase to maintain performance near $71 \%$ correct (Wetherill and Levitt, 1965). This task encouraged subjects to fixate the correct eye position throughout the duration of each run. We also monitored eye position in three subjects for accurate fixation with an infrared eye tracker (see Eye position measurements).

fMRI time series preprocessing. Data for each functional scan were corrected for head motion (Nestares and Heeger, 2000), linearly detrended, high-pass filtered with a cutoff frequency of $0.01 \mathrm{~Hz}$ to remove lowfrequency drift, and converted to percentage signal change by dividing each voxel's time series by its mean image intensity. The first half-cycle (12 s) of the time series was discarded, leaving 10 full cycles remaining, to allow both the longitudinal magnetization and the hemodynamics to reach steady state. All data analysis steps were performed with custom software (mrTools, http://www.cns.nyu.edu/heegerlab/?page= software) written in Matlab (The MathWorks).

$f M R I$ response amplitude and phase. The size and relative timing of the cortical response to each cycle of the rotating wedge stimulus was computed using a short-time Fourier transform. The time series of image intensities from each voxel in each run was computed by subdividing the time series into 10 nonoverlapping segments corresponding to each $24 \mathrm{~s}$ cycle (or repeat) of the stimulus. The amplitude and phase corresponding to the response at the stimulus frequency $(1 / 24 \mathrm{~s})$ was computed from the Fourier transform of each segment. This yielded a distribution of 10 response amplitudes and phases for each run. Response amplitude quantified the size of the evoked response in units of percentage change relative to the mean (over time) image intensity of each voxel. Response phases quantified the polar angle location of the wedge stimulus that evoked the largest response (Engel et al., 1994, 1997; Sereno et al., 1995; DeYoe et al., 1996).

The reliability of the responses from each voxel and each run was quantified as the coherence between the full time series (not segmented into the individual cycles/repeats) and the best-fitting sinusoid. Coherence is the ratio between the power at the stimulus frequency (1/24s) and the square root of the sum of squares of the power at all frequencies. High 
coherence values indicated that the response amplitudes and phases were reliable across all cycles of the stimulus.

Decoding eye position. A multivoxel pattern classification analysis was used to determine whether eye positions could be decoded from the spatial patterns (across voxels) of fMRI response amplitudes and/or phases. The analysis was performed separately for each visual cortical area region of interest (ROI; see Defining regions of interest). The measured response amplitudes from a single visual area in a single subject and session formed an $m \times n$ matrix, with $m$ being the number of voxels in the ROI and $n$ being the number of repeated measurements. In this experiment, there were a total of 160 repeated measurements per session ( $n=10$ repeats of the stimulus per run $\times 2$ runs per eye position $\times 8$ eye positions), and a total of 10 sessions ( 5 subjects, 2 sessions each). In principle, sessions could have been combined in two ways. First, we could have concatenated the sessions, leaving the number of voxels $(m)$ the same, but increasing the number of measurements $(n)$. This would have required precise registration across sessions. Second, we could have stacked the datasets across voxels, yielding a matrix of size $M \times n$ where $M$ was the total number of voxels summed across subjects and sessions. We chose the second approach because it did not depend critically on precise, subvoxel alignment across sessions.

Dimensionality reduction. For the analyses in which we pooled data across subjects and sessions (see Figs. 4A, 5, 6) we used principal components analysis (PCA) to reduce the dimensionality of $M$ from the total number of voxels across subjects and sessions $(\sim 3000$ in V1) to a smaller number of principal component scores (Pereira et al., 2008; Pereira et al., 2009; Brouwer and Heeger, 2009). The number of voxels differed between ROIs because of differences in the physical sizes of the visual cortical areas. After PCA, the dimensionality was set to the number of components needed to explain $68 \%(\sim 2$ SDs $)$ of the variance within a particular ROI, ignoring the remaining components. The resulting number of components was typically two orders of magnitude smaller than the original dimensionality (number of voxels) for each ROI.

Classification was performed with a eight-way maximum likelihood classifier, implemented by the Matlab function "classify" with the option "diagLinear." The distributed spatial pattern of response amplitudes (or phases) for each eye position can be described as a point in a multidimensional space where each dimension represents responses from a voxel (or principal component). Accurate decoding is possible when the responses measured for each eye position form distinct clusters within the space. The maximum likelihood classifier optimally separates trials belonging to each of the eight different eye positions, if the response variability in each voxel is normally distributed and statistically independent across voxels. Because the number of voxels, $M$, was large relative to the number of repeated measurements, $n$, the computed covariance matrix would have been a poor estimate of the real covariance. Even after dimensionality reduction with PCA, the number of elements of the covariance matrix ( $\sim 900$ in V1) was much larger than the number of repeated measurements (160). This would have made the performance of the classifier unstable, as it would have relied on inversion of this covariation matrix. We therefore ignored covariances between voxels (or principal components) and modeled the responses as being statistically independent. Although noise was likely spatially correlated, the independence assumption, if anything, was conservative; including accurate estimates of the covariances (if available) would have improved the decoding accuracies.

Decoding accuracy was computed using a leave-one-out crossvalidation procedure. We calculated (or "trained") the classifier on a set of exemplars (response amplitude or phase measurements, each corresponding to a single cycle of periodic stimulation), while excluding from training (i.e., leaving out) a single "test" exemplar, as well as the exemplars immediately preceding and following the test exemplar (i.e., so that the "test" exemplar was measured at a time $>24 \mathrm{~s}$ from each the "training" exemplars). We then tested whether the left-out test exemplar was accurately assigned to the proper eye position. The training and decoding process was repeated for each of the available exemplars. Decoding accuracy was determined as the proportion of exemplars that the classifier was able to correctly assign to one of the eight eye positions. We also computed decoding accuracy using other standard cross-validation proce- dures (e.g., $k$-fold cross-validation) and found qualitatively similar results that supported the same conclusions.

We performed a nonparametric permutation test to determine the statistical significance of decoding accuracy. For each visual cortical area, we randomly shuffled the eye position labels and computed the decoding accuracy (as described above). Repeating this 10,000 times yielded a distribution of decoding accuracies, according to the null hypothesis that eye position could not be classified. Decoding accuracies computed with the correctly labeled eye positions were then considered statistically significant when decoding accuracy was higher than the 95th percentile of the null distribution $(p<0.05,1$-tailed permutation test, Bonferroni corrected for multiple comparisons across visual areas).

Reconstructing eye position. A complementary analysis tested whether a planar model of gain fields would accurately reconstruct eye position from the spatially distributed patterns of voxel responses. We adopted an approach based on a "forward model", similar to that used previously to reconstruct stimulus color (Brouwer and Heeger, 2009) and stimulus orientation (Brouwer and Heeger, 2011), and to identify which of a large number of natural images was presented (Kay et al., 2008). The forward model in our case assumed that each voxel contained a large number of gain-modulated neurons, and that the gain field of each neuron was planar, $g=a x+b y+c$, where $g$ is gain, $x$ is horizontal eye position, $y$ is vertical eye position, and $a, b$, and $c$ determine the plane. We further assumed that the response of a voxel was proportional to the summed responses of all the neurons in that voxel, and that all of the neurons in a voxel shared the same receptive field (center) location.

It follows from this forward model that the response amplitude of each voxel can be characterized by a planar gain field. Because the stimulus rotations were periodic, the response time course of an individual neuron can be approximated as a sinusoid in the following equation: $r_{k}(t)=g_{k}$ $\sin \left(\omega t+\phi_{k}\right)$, where $r_{k}$ is the response of the $k^{\text {th }}$ neuron in voxel $m, \omega$ is the frequency of the stimulus rotations $(1 / 24 \mathrm{~Hz}), g_{k}$ is the gain that determines the amplitude of modulation of the neuron's response as the moving wedge passes through the neurons receptive field, and $\varphi_{k}$ is the phase of the neuron's response that depends on its receptive field location. The response gain in turn depends on eye position according to the following equation: $r_{k}(t)=\left(a_{k} x+b_{k} y+c_{k}\right) \sin \left(\omega t+\phi_{k}\right)$.

If all neurons in a voxel share the same receptive field center, then we can drop the subscript on the response phase, $\varphi$, and summing across neurons in the voxel gives the following:

$$
\begin{aligned}
R_{m}(t) & =\sum\left(a_{k} x+b_{k} y+c_{k}\right) \sin (\omega t+\phi) \\
& =\left[\sum a_{k} x+\sum b_{k} y+\sum c_{k}\right] \sin (\omega t+\phi) \\
& =\left(A_{m} x+B_{m} y+C_{m}\right) \sin (\omega t+\phi) .
\end{aligned}
$$

Hence, the response of the voxel exhibits a planar gain field. We determined through simulations that even with isotropic scatter in receptive field locations, the summed responses of neurons with planar gain fields yields a voxel with a planar gain field.

The eye position reconstruction analysis proceeded as follows. The response amplitudes for any one repeat/cycle of the stimulus can be rewritten in matrix notation for all voxels (or principal components) in an ROI according to the following equation:

$$
\vec{r}=\mathrm{Wx},
$$

where $\overrightarrow{\boldsymbol{r}}$ is a vector of voxel response amplitudes, $\vec{x}=(x, y, 1)^{T}$ is eye position, and $\mathbf{W}$ is a weight matrix with each row containing $A_{\mathrm{m}}, B_{\mathrm{m}}$, and $C_{\mathrm{m}}$. Let $m$ be the number of voxels (or principal components), and $n$ be the number of repeated measurements (i.e., 10 repeats per run $\times 8$ eye positions $\times 2$ runs per eye position). A matrix of response amplitudes corresponding to all of the repeated measurements $(\mathbf{R}, m \times n)$ can be related to a matrix of eye positions $(\mathbf{X}, 3 \times n)$ by a weight matrix $(\mathbf{W}, m \times$ 3): $\mathbf{R}=\mathbf{W X}$.

The measured response amplitudes were divided up into testing and training sets $\left(\mathbf{R}_{1}\right.$ and $\left.\mathbf{R}_{2}\right)$ according to the same partitioning protocol as for the classification analysis. Specifically, $\mathbf{R}_{1}$ consisted of an $m \times n$ matrix in which $n$ was the number of response amplitudes from each 
cycle of the stimulus at each eye position, minus a single left-out exemplar amplitude $\left(\mathbf{R}_{2}\right)$ and the exemplar amplitudes measured just before and just after $\mathbf{R}_{2}$. The training set was used to estimate weights, $\mathbf{W}$, using linear regression:

$$
\hat{\mathbf{W}}^{\mathrm{T}}=\mathbf{R}_{1} \mathbf{X}_{1}^{\mathrm{T}}\left(\mathbf{X}_{1} \mathbf{X}_{1}^{\mathrm{T}}\right)^{-1}
$$

The eye positions for the test data $\left(X_{2}\right)$ were then estimated using the estimated weights $(\hat{\mathbf{W}})$ :

$$
\hat{\mathbf{X}}_{2}=\left(\hat{\mathbf{W}}^{\mathrm{T}} \hat{\mathbf{W}}\right)^{-1} \hat{\mathbf{W}}^{\mathrm{T}} \mathbf{R}_{2}
$$

Because $\mathbf{R}_{2}$ consisted of a single exemplar response amplitude, the reconstructed eye position for that exemplar $\left(\mathbf{X}_{2}\right)$ consisted of a single screen position coordinate. Iterating over each left-out exemplar yielded a distribution of reconstructed screen coordinates. This procedure would have resulted in accurate reconstruction of eye positions for the test data if (1) the distributed pattern of responses across voxels contained information that differentiated the different eye position conditions; and (2) the differences in response amplitudes across the eight fixations were well-modeled by a plane. We measured the accuracy of reconstruction by computing the distance between the reconstructed and actual eye positions. Statistical significance of the reconstruction accuracies was determined using a one-tailed permutation test similar to that used to compute statistical significance of the classification analysis (see Multivoxel pattern classification).

A further test of the planar gain field model was to remove from the training set all the data corresponding to one eye position, so that it was novel during testing. We again separated the data into test and training sets $\left(\mathbf{R}_{1}\right.$ and $\left.\mathbf{R}_{2}\right)$. The training set consisted of all measured responses evoked by seven out of the eight eye positions. The test set consisted of all measured responses evoked by the one left-out eye position. We then trained the forward model (i.e., estimated the weights) on the training data and performed the reconstruction of the left-out eye position in the test data. On each iteration of the reconstruction analysis, the reconstructed eye position $\left(\mathbf{X}_{2}\right)$ consisted of a set of 20 screen coordinates, one for each cycle of the stimulus at the eye position left out from training. This process was repeated for all eight eye positions, leaving one out at a time.

Defining ROIs. Visual cortical areas were defined using standard retinotopic mapping methods (Sereno et al., 1995; DeYoe et al., 1996; Engel et al., 1997; Larsson and Heeger, 2006). The radial and angular components of the retinotopic map were displayed on flattened representations of the cortical surface. For each observer, a high-resolution anatomical volume (see MRI acquisition) was segmented and computationally flattened using FreeSurfer. Visual area boundaries were drawn by hand on the flat maps, following published conventions (Larsson and Heeger, 2006), and the corresponding gray matter coordinates were recorded. There is some controversy over the exact definition of human V4 and the area just anterior to it (Tootell and Hadjikhani, 2001; Brewer et al., 2005; Hansen et al., 2007). We adopted the conventions proposed by Wandell et al. (2005) in defining human V4 as the full hemifield representation in the ventral occipital lobe just anterior to ventral V3. Using data acquired in a separate scanning session, area MT was defined as an area in or near the dorsal/posterior limb of the inferior temporal sulcus that (1) responded more strongly to coherently moving dots relative to static dots (Tootell et al., 1995), setting it apart from neighboring areas LO1 and LO2 (Larsson and Heeger, 2006); and (2) responded to the rotating wedge stimulus in the retinotopic mapping experiment, setting it apart from area MST, which has larger receptive fields that are not strongly activated by the rotating wedge stimulus (Huk et al., 2002). VO1 and $\mathrm{VO} 2$ were defined using the conventions described by Brewer et al. (2005). Voxel responses from VO1 and VO2, and from LO1 and LO2, were pooled for the classification analysis.

Voxel selection. We used two criteria to select a subset of voxels within each of these visual cortical areas. First, we selected voxels that fell within a $4^{\circ}$ radius of the fovea, as determined from the radial component of the retinotopic map (see Defining ROIs). Second, we selected voxels that were reliably driven by the stimulus (coherence, $>0.4$ ) in at least one run of the main (gain field) experiment. The analysis was repeated without the coherence threshold; the results of this analysis supported the same conclusions although the decoding accuracies were lower because less reliable voxels were included.

MRI acquisition. Anatomical and functional MRI data were acquired on a Siemens 3T Allegra head-only scanner using a transmit head coil (NM-011, Nova Medical) and a four-channel phased array receive surface coil (NMSC-021, Nova Medical). Functional scans were acquired with gradient recalled echo-planar imaging to measure blood oxygen level-dependent changes in image intensity (Ogawa et al., 1990). Functional imaging was conducted with 24 slices placed perpendicular to the calcarine sulcus (TR, $1500 \mathrm{~s}$; TE, $30 \mathrm{~ms}$; flip angle, $75^{\circ}$; voxel size, $2 \times 2 \times$ $2 \mathrm{~mm} ; 80 \times 120$ grid size). A T1-weighted magnetization-prepared rapid gradient echo anatomical scan was acquired in each scanning session with the same slice prescriptions as the functional images (TR, $1530 \mathrm{~ms}$; TE, $3.8 \mathrm{~ms}$; flip angle, $8^{\circ}$; voxel size, $1 \times 1 \times 2.5 \mathrm{~mm}$; voxel grid, $256 \times$ 160 ). This anatomical scan was aligned using a robust image registration algorithm (Nestares and Heeger, 2000) to a high-resolution, full-brain anatomical volume, which was acquired in a separate session (TR, 2500 $\mathrm{ms}$; TE, $3.93 \mathrm{~ms}$; flip angle, $8^{\circ}$; voxel size, $1 \times 1 \times 1 \mathrm{~mm}$; voxel grid, $256 \times$ 256). The full-brain anatomical volume was used for both registration across scanning sessions and for gray matter segmentation and cortical flattening.

Visual stimulus presentation. Visual stimuli were presented with an LCD projector (LC-XG100, Eiki) with a pixel resolution of $1024 \times 768$. Subjects viewed the image from the LCD projector on a rear projection screen placed inside the bore of the magnet at a distance of $57 \mathrm{~cm}$, yielding a field of view of $29 \times 22^{\circ}$. The display was linearized using a Photo Research PR650 SpectraColorimeter. Stimuli were generated using Matlab with MGL (http://justingardner.net/mgl). The inside surface of the head coil and scanner bore were lined with black felt to eliminate stray light reflection.

Eye position measurements. It was critical that subjects fixated the appropriate location for the duration of each $4.2 \mathrm{~min}$ fMRI scanning run. It is unlikely that subjects made large eye movement errors during the main fMRI experiment, for three reasons. First, all subjects had extensive prior experience in psychophysical and functional imaging experiments. Second, the demanding fixation task performed during scanning required accurate fixation. Finally, an MRI-compatible camera-based eye tracker (ASL Model 504, Applied Science Laboratories) was used to visually ensure that subjects were alert and fixating the appropriate location. Nonetheless, because this experiment involved periods of extended fixation ( $4.2 \mathrm{~min}$ per run) in the presence of a potent visual stimulus (inward/outward radial dot motion), it is conceivable that there were small, involuntary eye movements or fixation errors. Small errors could potentially create an artifact if they varied systematically across the eight intended fixation positions.

To test this possibility, we monitored eye position during a replication experiment. Each of three subjects ( 2 of whom also participated in the main experiment) were scanned in a protocol identical to the main experiment, except for the following four factors. 1) Eye position was monitored during fMRI scanning with a high-resolution eyetracker (MRcompatible EyeLink 1000). 2) Subjects simply fixated a cross rather than perform the demanding attention task used in the main experiment. The attention task was not required to ensure stable fixation because we were able to confirm accurate fixation by using the eye position recordings. 3 ) A smaller vertical range of intended fixation positions was tested $\left( \pm 9^{\circ}\right.$ horizontal and $\pm 3^{\circ}$ vertical) to accommodate the reduced field of view caused by the presence of the eyetracker at the bottom of the scanner bore. 4) An eight-channel receive coil (NMSC-071, Nova Medical) was used, rather than the four-channel coil.

\section{Results}

We measured fMRI responses while subjects maintained fixation at one of eight screen positions (Fig. 1A). The stimulus consisted of a circular array of static dots. One-quarter of the dots formed a motion-defined wedge while the rest of the dots remained stationary. The polar angle of the wedge rotated about fixation, similar in fashion to the rotating wedge stimulus used in standard 
A

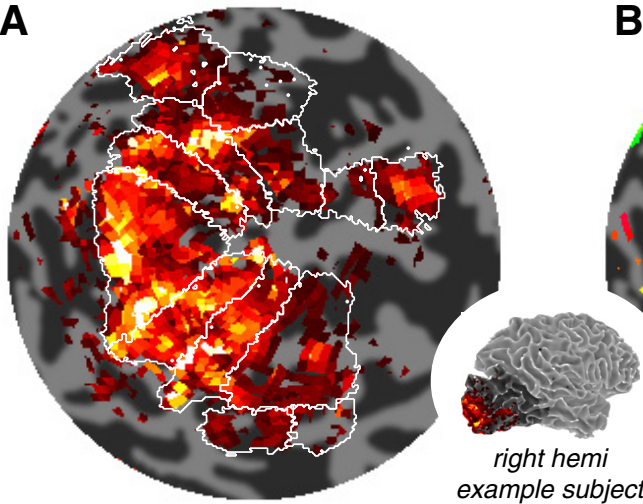

Average response amplitude

(\% change image intensity)

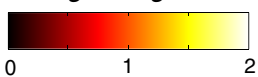

B

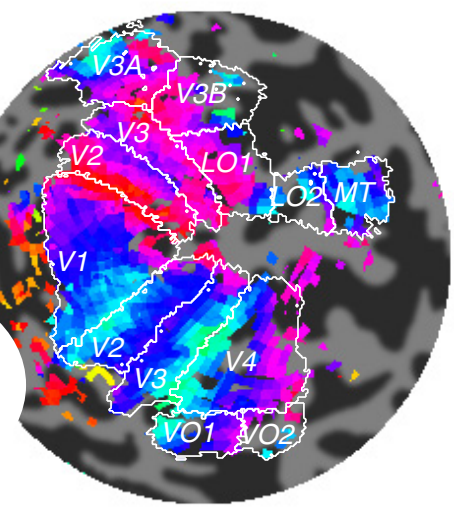

Average response phase (radians)

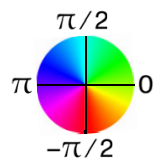

Figure 2. Cortical responses to the motion-defined rotating wedge stimulus (single subject, single session, averaged across the eight eye positions). Time series from each voxel were fit with sinusoids with period equal to that of the stimulus rotation. $\boldsymbol{A}$, Amplitude of the best-fitting sinusoid indicates the amplitude of the cortical response. $\boldsymbol{B}$, Phase angle of the best-fitting sinusoid indicates the stimulus position that elicited the largest response. Responses have been projected onto a computationally flattened representation of the subject's right hemisphere. White lines indicate visual area boundaries defined in an independent retinotopic mapping session.

retinotopic mapping experiments. We measured the response amplitudes (Fig. 2A) and response phases (Fig. 2B) for each voxel in visual cortex that responded to the rotating wedge stimulus.

We illustrate our main results with responses measured from a single voxel in visual area V3A (Fig. 3); the responses from this voxel demonstrate three features characteristic of the full dataset and consistent with a retinotopic visual representation in which eye position modulates the gain of visual responses. First, the response time courses (Fig. 3, plot symbols) at each eye position were well fit by sinusoids (Fig. 3, black curves) with frequency equal to that of the stimulus rotation. Second, the sizes of the visual responses, as measured by the amplitudes of the bestfitting sinusoids, changed as a function of eye position (Fig. 3, center). For this particular voxel, the response amplitude was largest when the eyes were up and to the right and it was smallest when the eyes were down and to the left. This change in response amplitude indicated that eye position modulated the gain of the visual response. For this voxel, the dependence of response gain on eye position was well approximated by a plane. Third, the spatial selectivity of the responses, as measured by the phase of the best-fitting sinusoid, did not vary with eye position. This invariance indicates that this voxel was selective for the location of the stimulus on the retina rather than, for example, the location of the stimulus on the screen.

\section{Decoding eye position from response amplitudes}

There was enough information in the response amplitudes to decode eye position. We trained a maximum-likelihood classifier to identify eye position based on the spatial patterns of response amplitudes across voxels. We report results both combined (pooled) across subjects and sessions (Fig. 4A), and computed individually for each subject and then averaged (Fig. 4B). Decoding of eye position was significantly above chance for all eight eye positions, for several visual cortical areas including $\mathrm{V} 1, \mathrm{~V} 2, \mathrm{~V} 3$, V3A, and MT (Fig. 4A, dark bars). The results from the classifier

analysis indicated that changes in static eye position evoked consistent and reliable changes in visually driven response amplitudes. A complementary ANOVA confirmed that a large proportion of voxels in each of several visual cortical areas (V1, V2, V3, V3A, V7, LO, and MT) exhibited statistically significant modulations in response amplitudes across the eight eye positions (Fig. 4C, dark bars).

We examined the frequency with which the classifier assigned each of the eight actual eye positions to each of the eight potential eye positions by making a confusion matrix in which large values along the diagonal of the matrix indicated accurate decoding (Fig. 5A). Decoding accuracy was statistically significant $(p<$ 0.05 , permutation test) for each of the eight eye positions (tested individually), for each of the five visual areas that yielded the highest overall decoding accuracies (V1, V2, V3, V3A, MT). High values along the diagonal of the confusion matrix demonstrated that the spatial patterns of fMRI response amplitudes encoded information about all of the individual eye positions, not just differences between left versus right eye positions, nor up versus down, nor that the overall decoding accuracy was driven by just one or a few of the eye positions. When the classifier failed, it usually classified the responses as being evoked by an adjacent eye position, and rarely confused eye positions from opposite sides of the screen (Fig. 5B). For comparison, we also examined the confusion matrix from V4, which did not exhibit significant eye position classification, and consequently had low values along the diagonal (Fig. $5 C, D$ ).

Accurate decoding depended on information available in the spatially distributed pattern of response amplitudes, not differences in the mean response amplitudes averaged across voxels in each visual area ROI. To test this possibility, we computed the mean response amplitude, averaged across voxels in each ROI, separately for each observer. We found no instances of significant differences between the mean amplitudes for different eye positions. We attempted to decode eye position based on the mean responses in each ROI using the same cross-validation procedure used to classify eye position from the spatial patterns of response amplitudes. Decoding accuracies based on these mean responses were not significantly different from chance. This suggests that the eye-position dependence of cortical activity was spatially heterogeneous across voxels, analogous with the conclusion from electrophysiological studies that gain fields differ across the population of neurons.

\section{Decoding eye position from response phases}

Our decoding results thus far support the hypothesis that visual cortical areas represent stimulus locations in a retinotopic reference frame, modulated by gain fields. A theoretical alternative is that some or all of these visual areas, or subsets of voxels within these cortical areas, represent stimuli in a "spatiotopic" reference frame, linked to the location of stimuli in space (i.e., screen coordinates), independent of eye position (d'Avossa et al., 2007). If, indeed, the visuotopic maps in any of the visual areas were spatiotopic instead of retinotopic, then this would have predicted 
changes in response amplitudes with changes in eye position. For example, consider a voxel from a spatiotopic map that responds more strongly when stimuli are presented on the left half of the screen, regardless of eye position. In our experimental protocol, stimuli were presented on the left half of the screen only when the fixation point was on the left half of the screen. Hence, eye positions on the left would have evoked larger response amplitudes than eye positions on the right.

If the cortical maps were spatiotopic, then the response phases would have also varied systematically with eye position. For example, consider a voxel from a spatiotopic map that responds more strongly when stimuli are presented at the center of the screen, regardless of eye position. For left eye positions, this voxel's response would have been largest when the rotating wedge stimulus was on the right. For right eye positions, this voxel's response would have been largest when the wedge was on the left (i.e., $180^{\circ}$ out of phase).

There was no evidence, however, that eye position affected response phases (Fig. $4 A, B$, light bars). We attempted to train the classifier to discriminate eye positions based on the spatial patterns of response phases, using exactly the same approach we used to discriminate eye positions based on response amplitudes. For none of the visual areas was decoding accuracy significantly above chance (i.e., the classifier failed to discriminate eye positions based on response phases). A complementary ANOVA confirmed that very few (if any) of the voxels in each visual cortical area exhibited significant modulation in response phases across the eight eye positions (Fig. $4 C$, light bars). None of the visual areas exhibited an above-chance percentage of voxels with significant phase modulation.

\section{Reconstructing eye position from gain fields}

We tested whether the gain fields were well characterized by a planar function of eye position, analogous to the planar functions described for single neurons in the lateral intraparietal area (Andersen et al., 1990). We adopted an approach based on a "forward model," which is conceptually similar to that used previously to reconstruct stimulus color (Brouwer and Heeger, 2009) and orientation (Brouwer and Heeger, 2011), and to identify which of a large number of natural images was presented (Kay et al., 2008), but used here to reconstruct eye position from the spatially distributed patterns of voxel responses. The reconstruction analysis was conceptually related to the classification analysis in that it took advantage of any reliable biases distributed across the population of voxels within a given visual area. However, unlike the classification analysis, the reconstruction analysis assumed an underlying function to the pattern of responses, which in this case was a planar gain field.

The planar model provided a reasonably good fit to the measured response amplitudes in individual voxels. The best-fitting
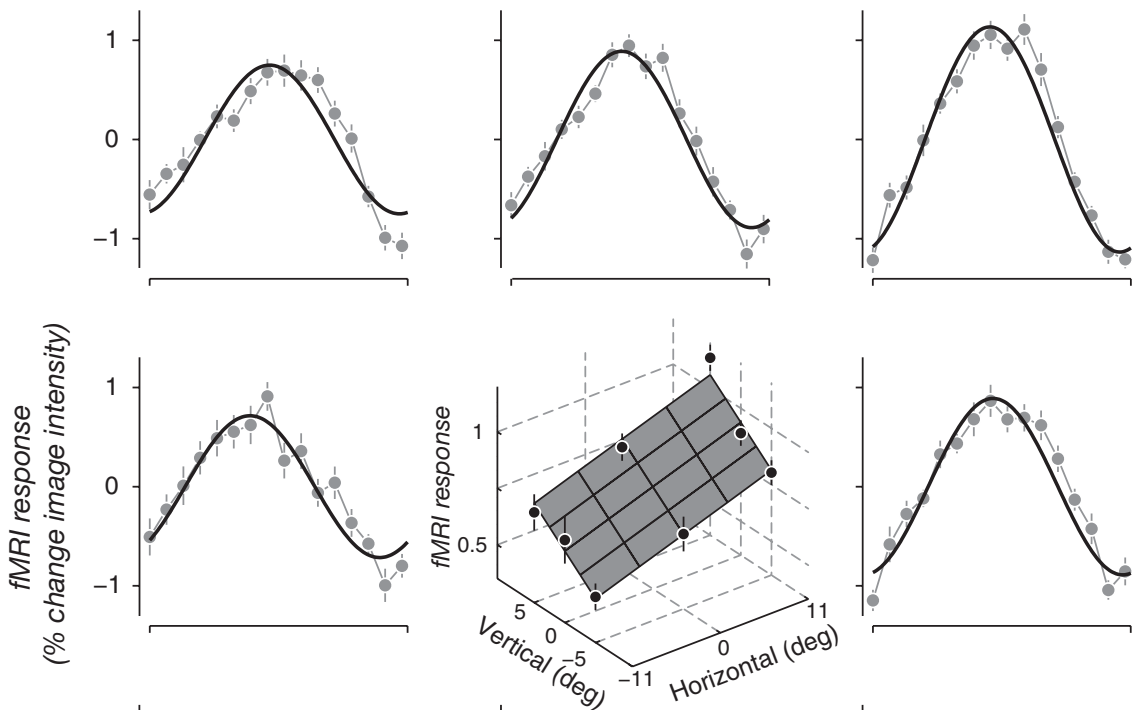

Figure 3. Eye position modulates the amplitude, but not the phase, of visual responses. Time series plots show the cortical responses from a single voxel in area V3A for each of the eight eye positions tested in a single session. Gray dots and vertical lines a dive fime series across 20 repeats of the stimulus at a particular eye position. Black curves subject fixated up and to the right $\left(+11^{\circ},+5^{\circ}\right)$ and was smallest when the subject fixated down and to the left $\left(-11^{\circ},-5^{\circ}\right)$. with eye position was well fit by a plane with a particular angle and slope (center). The spatial selectivity of the responses, as (1)

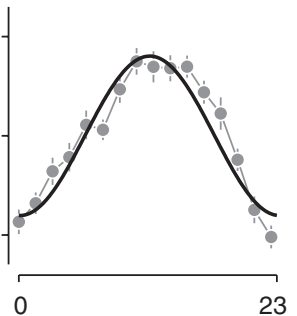

Time (seconds) 
A

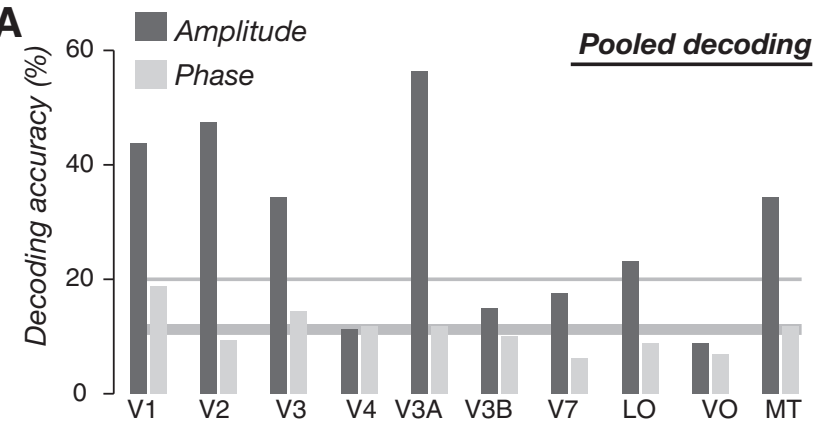

B

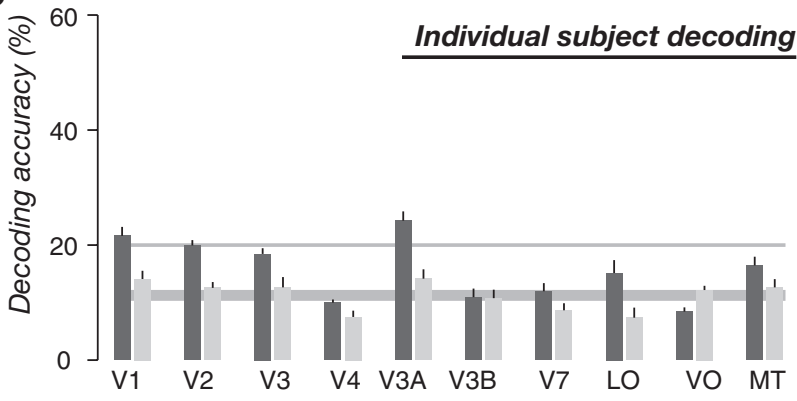

C

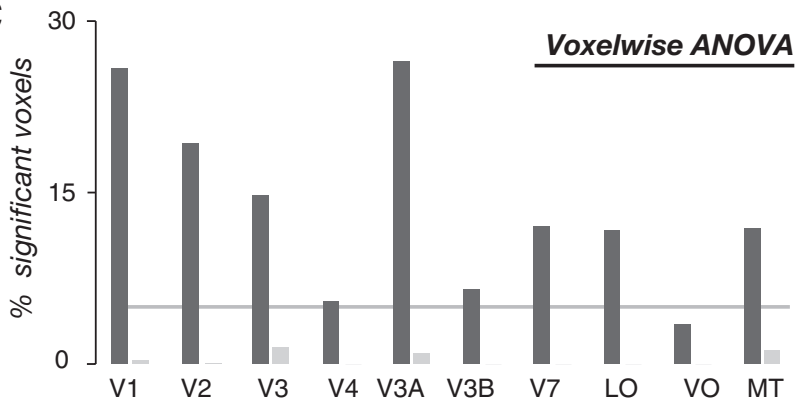

Figure 4. Decoding eye position with the amplitude, but not the phase, of visually evoked activity. $\boldsymbol{A}$, Eye position decoding, based on response amplitude (dark bars) and response phase (light bars), pooled across subjects and sessions. Thick and thin horizontal lines indicate decoding accuracies that would have been achieved by chance: 50 th and 99th percentile, respectively, of the null distribution, according to the null hypothesis that eye position could not be classified. $\boldsymbol{B}$, Individual subject decoding accuracy, computed individually for each subject and then averaged across subjects. Error bars indicate SEM across subjects. C, Percentage of voxels in each cortical area exhibiting significant (false discovery rate, $<0.05$ ) modulation across eye position. Horizontal line indicates the percentage of significant voxels that would be expected according to the null hypothesis that responses did not vary across eye positions.

however, might have evoked responses in voxels corresponding to peripheral visual field locations. Such responses would differ across the different eye positions. For example, when the eye position and stimulus aperture were on the left side of the screen, there might have been a reflection of the stimulus from the left side of the scanner bore, whereas this reflection would have been on the right when fixating on the right. Such differential responses would have enabled high decoding accuracies, not because of gain fields, but simply because the visual stimulus would have been different for different eye positions.

The experiment was designed to minimize the possibility that any such artifacts confounded the interpretation of our results. First, the inside surface of the head coil and scanner bore were lined with black felt to minimize stray light reflection. Second, the stimulus was designed to minimize the possibility of such artifacts. Specifically, we used a motiondefined wedge in which the luminance and contrast were equal within the wedge and the rest of the stimulus aperture. If there had been any differences in contrast or luminance (as is the case for a more conventional retinotopic mapping stimulus in which the wedge contrasts highly against a uniform gray background), then any residual reflection would have evoked activity in voxels corresponding to peripheral visual field locations. Such reflections would have modulated periodically over time with the stimulus rotations.

\section{Influence of fixational eye movements}

It was important that subjects fixated the appropriate location. The fixation task ensured reasonably accurate fixation, but did not preclude the possibility of small eye position errors. To address this issue, three subjects participated in a replication of the main fMRI experiment with concurrent, high-quality eye tracking.

Eye position could be reliably decoded from each of the five visual areas that yielded the highest overall decoding accuracies in the main experiment, replicating our main result. We pooled the voxel responses across the three sessions in the replication experiment for which we verified accurate fixation. Decoding accuracies were as follows: V1, 31\%; V2, 35\%; V3, 38\%; V3A, 32\%; MT, $32 \%$ ( $p<0.05$, permutation test, Bonferroni corrected for number of ROIs). Decoding accuracy was also significant $(p<0.05$, permutation test, Bonferroni corrected) in each of the three individual (unpooled) subjects in V2 $(24 \pm 2 \%)$, V3 (26 $\pm 7 \%)$, and V3A $(24 \pm 3 \%)$, and in two out of three subjects in V1 $(20 \pm$ $2 \%)$ and MT (19 $\pm 3 \%)$.

Subjects were able to fixate reliably while viewing the motion stimulus. Large $\left(>1^{\circ}\right)$ saccades away from the fixation cross were rare, occurring typically once (if at all) in a $4.2 \mathrm{~min}$ run (Fig. 7A). Nevertheless, it is conceivable that small, involuntary eye movements (microsaccades) could have differed across the eight intended fixation positions. We detected microsaccades as small as $0.1^{\circ}$ (Engbert and Mergenthaler, 2006) that occurred during otherwise stable and accurate fixation (Fig. $7 B, C$ ). We then quantified microsaccade amplitude, rate, and direction (Fig. $7 D-F$ ). We found no evidence for differences in any of these parameters across the eight intended fixation positions (not significant, permutation tests for each individual subject). Fixation position (extracted from periods between saccades) was slightly biased in the direction of stimulus angle (corresponding to the moving portion of the stimulus). The bias was small but sufficient to train a multivariate classifier to decode the angle of the stimulus based on the raw horizontal and vertical eye position traces (decoding accuracy, $15 \% ; p<0.05$, permutation test). Critically, the bias depended only on the angle of the stimulus, and did not vary across the eight intended fixation positions. To test this, we centered the eye position data by subtracting the coordinates of each intended fixation position and then used the horizontal and vertical eye position traces to train a multivariate classifier to discriminate the eight intended fixation positions (mean decoding accuracy, $12.5 \%$; not significant, permutation test). Various factors might have resulted in abovechance eye position decoding based on the eye traces, even with perfect calibration. For example, intended fixation position might have been decoded from the eye traces if subjects made systematic fixation errors that depended on intended fixation position, or if microsaccade metrics (amplitude, direction, or rate) varied with intended fixation position). But there was no evidence for systematic deviations in eye position across the eight intended fixation positions, even though we 
Decoding in V3A
A

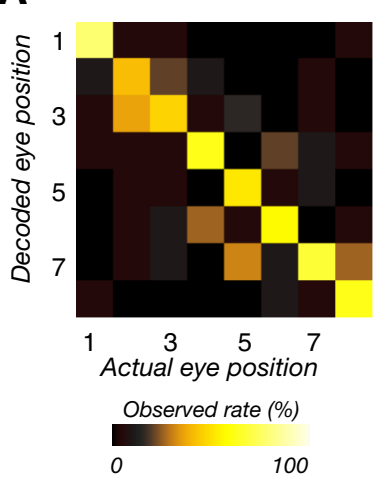

\section{C}
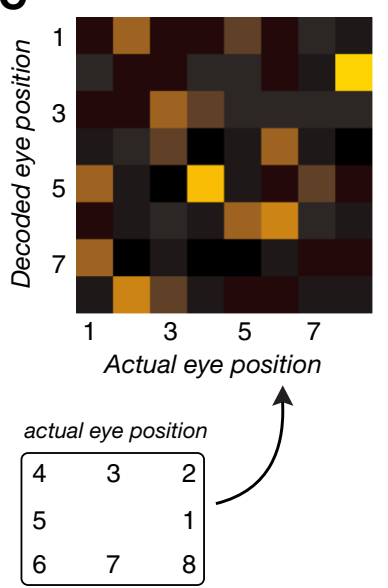

B

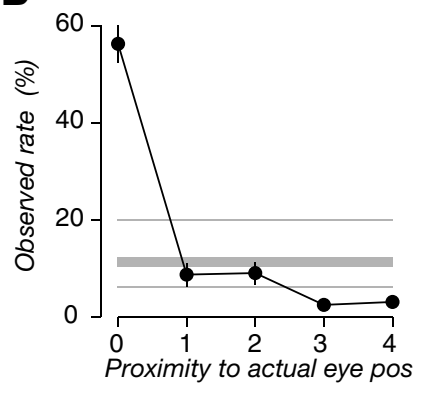

Decoding in V4
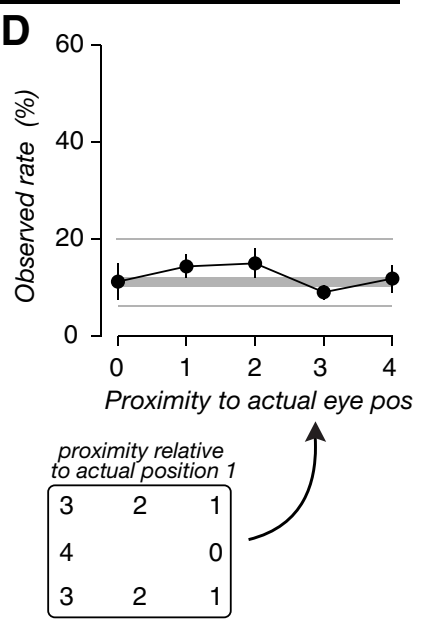

Figure 5. Eye position decoding. A, Confusion matrix for V3A, pooled across subjects and sessions. The confusion matrix depicts the percentage of repeats of each actual eye position $(x$-axis) for which each decoded eye position ( $y$-axis) was obtained. High values along the diagonal indicate accurate decoding. $\boldsymbol{B}$, Decoding pooled across actual eye positions. Data from the confusion matrix were averaged according to the proximity (inset below $\boldsymbol{D}$ ) to the actual eye positions. Thick horizontal line indicates the median of the null distribution. Two thin horizontal lines indicate the $2.5^{\text {th }}$ and $97.5^{\text {th }}$ percentile of the null distribution. C, D, Decoding accuracy in V4 (same conventions as in $\boldsymbol{A}$ and $\boldsymbol{B}$ ). Insets, Example of numbering of proximities of fixation positions for the case when the actual position was on the right at $+11,0$.

had sufficient statistical power to detect a small fixation bias with stimulus angle.

Finally, we asked if optokinetic nystagmus (OKN) differed across the eight intended fixation positions. The flow field of dots moving radially might have driven OKN, evident as periods of pursuit-like drifts in eye position aligned with stimulus motion (slow phase), followed by a rapid saccade-like return of the eyes back in the direction of fixation (fast phase). This would have potentially created an artifact if the magnitude of the OKN (the absolute displacement of the eyes) differed across the eight intended fixation positions (e.g., rightward drifts were smaller and leftward drifts were larger when fixation was on the right). We visually inspected the eye traces, but found no evidence for OKN (Fig. 7B). To test for the possibility of OKN and, in particular, differences in OKN across intended eye positions, we computed the SD of the radial component of eye position for each intended fixation position, and for each stimulus angle. There was no evidence for differences in eye position SD, either across intended fixation positions or stimulus angles. More importantly, there was no
A

10

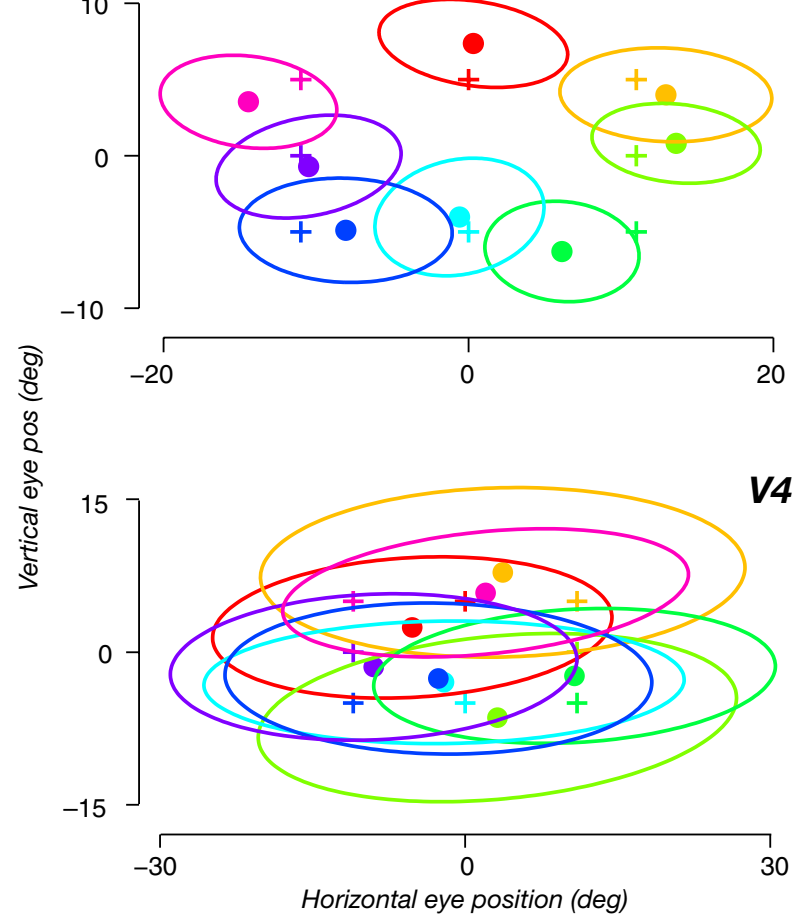

B

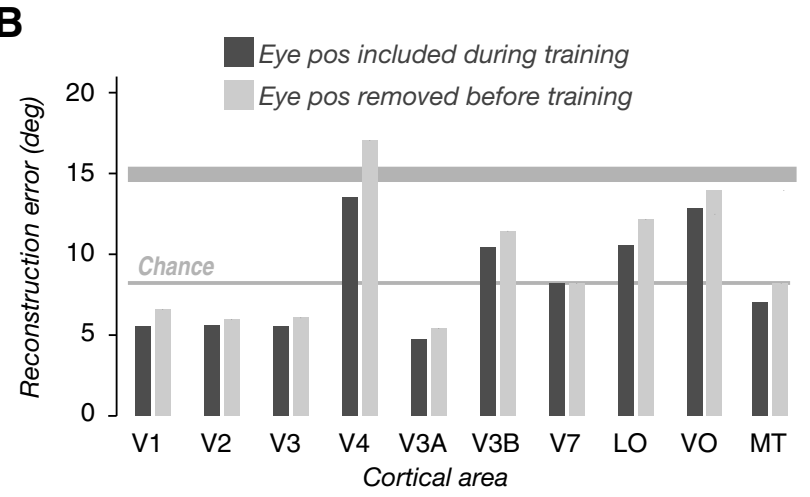

Figure 6. Planar gain field model enables accurate eye position reconstruction. $\boldsymbol{A}$, Reconstructed eye position from response amplitudes in V3A (top) and V4 (bottom). Data pooled across subjects and sessions. +, actual eye position; -, median reconstructed eye position; ellipses, 1 SD. B, Error in reconstructed eye position (median distance between reconstructed and actual eye position). Smaller values mean better reconstruction (opposite to Fig. 4). Dark bars, eye position reconstruction error when all eight eye positions were included in training to fit the planar gain field model. Light bars, eye position reconstruction error when the tested eye positions were excluded (one at a time) from training/fitting. Thick and thin horizontal gray lines indicate the error that would have been achieved by chance: 50th and 5th percentile, respectively, of the null distribution, according to the null hypothesis that eye position could not be reconstructed.

interaction, indicating that $\mathrm{OKN}$ did not vary with intended fixation position.

\section{Discussion}

The amplitude of visually driven cortical activity depended on eye position. The modulation of response amplitude varied across voxels so that eye position could be decoded from the spatial pattern of activity using a standard classifier, and eye position could be reconstructed using a forward model based on planar gain fields. The modulation of response amplitude in visual cortex was not due to an underlying spatiotopic representation, and was not influenced by confounds associated with unintended vi- 


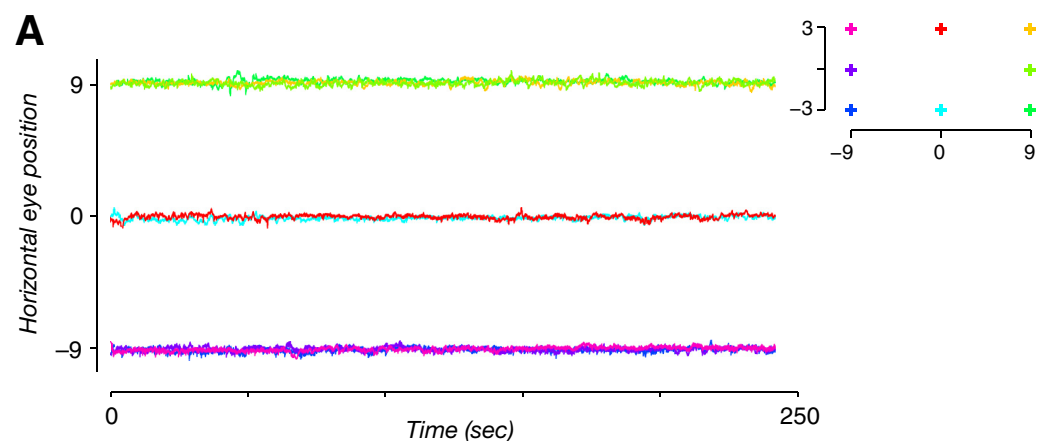

B
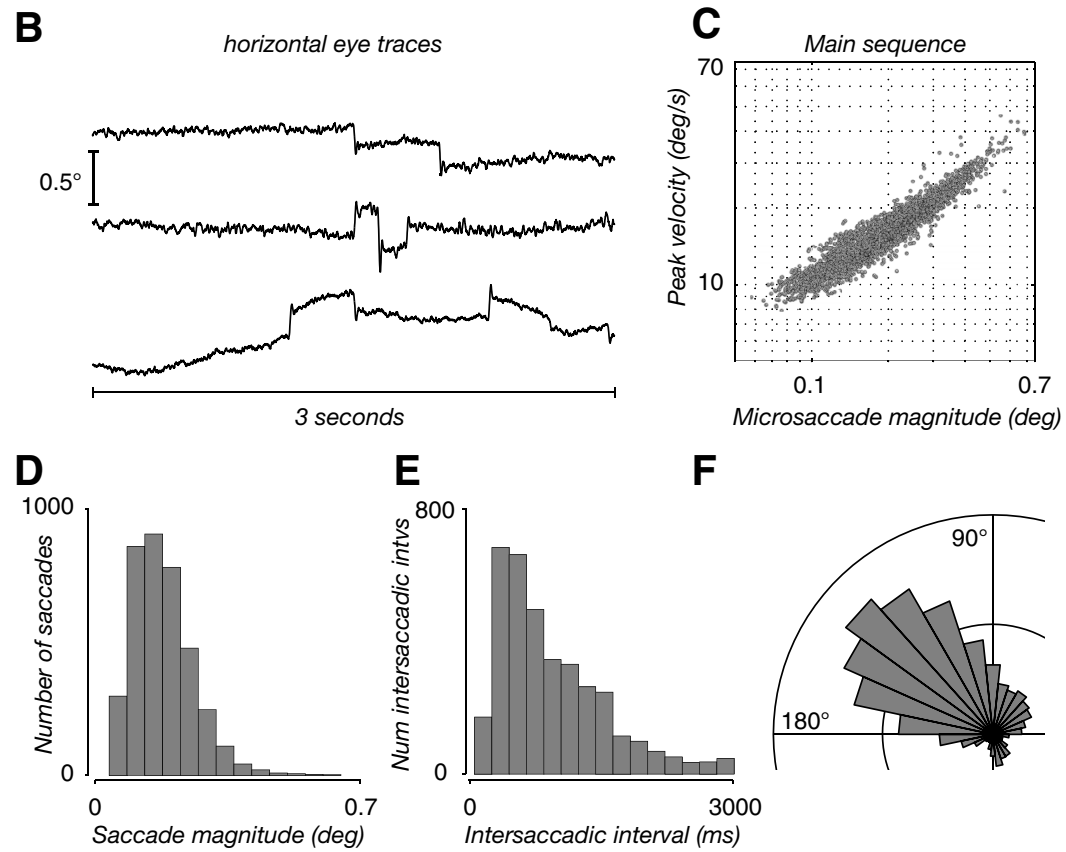

Figure 7. Eye position traces recorded during the replication fMRI experiment. $\boldsymbol{A}$, Horizontal eye position recorded during 4.2 min fMRI runs for each of the eight eye positions tested. $\boldsymbol{B}, 3 \mathrm{~s}$ excerpts of horizontal eye traces from three different intended fixation positions, illustrating the presence of microsaccades in the absence of OKN. C, Main sequence (peak velocity vs magnitude) for detected microsaccades exhibited the typical linear relationship in $\log$-log coordinates. D, Distribution of saccade magnitudes from one example subject. $\boldsymbol{E}$, Distribution of intersaccade intervals. $\boldsymbol{F}$, Distribution of saccade endpoints. There was no evidence for any differences, across intended fixation positions, in the distributions of saccade magnitudes, intersaccade intervals, and saccade endpoints.

sual stimulation in the periphery. We conclude that human visual cortex contains a retinotopic representation in which both eye position and stimulus location are represented.

Eye position gain fields are most often associated with parietal cortex, where the phenomenon was initially discovered and where gain fields have been most convincingly demonstrated (Andersen and Mountcastle, 1983). In the present study, we focused data acquisition and analysis on early visual cortex rather than on parietal cortex. There were two reasons for the decision. First, our previous study on visual reference frames (Gardner et al., 2008) demonstrated that all early visual cortical areas are retinotopic, and not spatiotopic. Hence, this set of cortical areas provided an ideal testbed for investigating eye position modulation of retinotopic visual responses. Second, we chose a particular stimulation protocol that avoided several potential confounds associated with investigating eye position effects with fMRI. The stimulus elicited motion-selective responses in cortical areas tuned to the polar angle of the stimulus within the central $4^{\circ}$ of visual space. In pilot data, we found this stimulus evoked strong responses in early visual areas, but not in parietal cortex, where receptive fields are typically larger (Schluppeck et al., 2005). While gain fields are not often associated with early visual cortex, single-unit recording studies have in fact demonstrated robust gain fields in each of the cortical areas for which we report having found evidence for them, including V1, V2, V3, V3A, and MT (Galletti and Battaglini, 1989; Galletti et al., 1995; Bremmer et al., 1998; Trotter and Celebrini, 1999; Rosenbluth and Allman, 2002; Sharma et al., 2003). Future fMRI studies using different visual stimulation protocols will be required to measure gain fields in higher-order areas in both the dorsal and ventral visual system.

Gain fields arise from the conjunction of visual input and information about static eye position. The pathways by which these two signals are integrated remains unknown. Gain fields in parietal cortex may arise from that region's dense anatomical connections with both early visual cortex and oculomotor zones in the superior colliculus and frontal lobe (Cavada and Goldman-Rakic, 1989; Andersen et al., 1990; Blatt et al., 1990; Morel and Bullier, 1990; Baizer et al., 1991). These pathways could provide the visual system with an efference copy of the eye-movement signal, and may thus play a role in establishing and dynamically updating gain fields in conjunction with changes in eye position. An alternative possibility is that eye position information is relayed to parietal cortex from primary somatosensory areas associated with eye muscle proprioception (Wang et al., 2007; Zhang et al., 2008; Xu et al., 2011). A somatosensory pathway may be too slow to be useful for the online control of visuomotor behavior, but could be important for computing the location of objects when the eyes are fixed, or for recalibrating a visuotopic map over periods of extended fixation. Because subjects held fixation over the course of each fMRI run, either of these pathways could account for eye position signals that we measured in the present experiment.

There is controversy concerning the reference frame(s) of spatial maps in visual cortex. Some previous studies have reported that some visual areas represent stimuli in a "spatiotopic" reference frame, linked to the location of stimuli in space (i.e., screen coordinates), independent of eye position (McKyton and Zohary, 2007; d'Avossa et al., 2007). But our results support the hypothesis that visual cortical areas represent stimulus locations in a retinotopic reference frame modulated by eye position (Gardner et al., 2008). There have been two proposals for how to reconcile these contradictory findings. First, the reference frames might be flexible such that the degree to which maps appear spatiotopic is task-dependent (Crespi et al., 2011). Responses may appear to be retinotopic when attention is directed to the fovea, and responses may appear spatiotopic when attention is not explicitly controlled (Crespi et al., 2011). Our results do not 
support the notion of flexible reference frames. We reliably decoded eye position from $\mathrm{AMRI}$ response amplitudes (but not from response phases) both when subjects were performing a demanding fixation task, and when they simply fixated, without explicit attentional control. Second, it has been argued that spatiotopic responses might arise from pooling the responses of neurons with diverse gain fields, within fMRI voxels (Pertzov et al., 2011). Our results do not support this conjecture either. We conclude that gain fields are superimposed on stable retinotopic maps, both of which were evident in fMRI responses. This observation indicates that pooling the responses of many gain-modulated neurons yields in a gainmodulated voxel response, not a spatiotopic map.

We have shown that the combination of gain fields and retinotopic responses provide sufficient information to localize objects in space despite variations in gaze. We conclude, as have others working at the level of single neurons (Andersen and Mountcastle, 1983), that gain fields make it unnecessary for the visual system to represent objects in an explicit spatiotopic frame of reference.

\section{References}

Andersen RA, Mountcastle VB (1983) The influence of the angle of gaze upon the excitability of the light-sensitive neurons of the posterior parietal cortex. J Neurosci 3:532-548. Medline

Andersen RA, Bracewell RM, Barash S, Gnadt JW, Fogassi L (1990) Eye position effects on visual, memory, and saccade-related activity in areas LIP and 7a of macaque. J Neurosci 10:1176-1196. Medline

Baizer JS, Ungerleider LG, Desimone R (1991) Organization of visual inputs to the inferior temporal and posterior parietal cortex in macaques. J Neurosci 11:168-190. Medline

Baker JT, Donoghue JP, Sanes JN (1999) Gaze direction modulates finger movement activation patterns in human cerebral cortex. J Neurosci 19: 10044-10052. Medline

Bédard P, Sanes JN (2009) Gaze and hand position effects on fingermovement-related human brain activation. J Neurophysiol 101:834-842. CrossRef Medline

Bédard P, Wu M, Sanes JN (2011) Brain activation related to combinations of gaze position, visual input, and goal-directed hand movements. Cereb Cortex 21:1273-1282. CrossRef Medline

Blatt GJ, Andersen RA, Stoner GR (1990) Visual receptive field organization and cortico-cortical connections of the lateral intraparietal area (area LIP) in the macaque. J Comp Neurol 299:421-445. CrossRef Medline

Boussaoud D, Barth TM, Wise SP (1993) Effects of gaze on apparent visual responses of frontal cortex neurons. Exp Brain Res 93:423-434. Medline

Boussaoud D, Jouffrais C, Bremmer F (1998) Eye position effects on the neuronal activity of dorsal premotor cortex in the macaque monkey. J Neurophysiol 80:1132-1150. Medline

Bremmer F, Pouget A, Hoffmann KP (1998) Eye position encoding in the macaque posterior parietal cortex. Eur J Neurosci 10:153-160. CrossRef Medline

Brewer AA, Liu J, Wade AR, Wandell BA (2005) Visual field maps and stimulus selectivity in human ventral occipital cortex. Nat Neurosci 8:11021109. CrossRef Medline

Brouwer GJ, Heeger DJ (2009) Decoding and reconstructing color from responses in human visual cortex. J Neurosci 29:13992-14003. CrossRef Medline

Brouwer GJ, Heeger DJ (2011) Cross-orientation suppression in human visual cortex. J Neurophysiol 106:2108-2119. CrossRef Medline

Cassanello CR, Ferrera VP (2007) Computing vector differences using a gain field-like mechanism in monkey frontal eye field. J Physiol 582:647664. CrossRef Medline

Cavada C, Goldman-Rakic PS (1989) Posterior parietal cortex in rhesus monkey: II. Evidence for segregated corticocortical networks linking sensory and limbic areas with the frontal lobe. J Comp Neurol 287:422-445. CrossRef Medline

Crespi S, Biagi L, d'Avossa G, Burr DC, Tosetti M, Morrone MC (2011) Spatiotopic coding of BOLD signal in human visual cortex depends on spatial attention. PLoS ONE 6:e21661. CrossRef Medline

d'Avossa G, Tosetti M, Crespi S, Biagi L, Burr DC, Morrone MC (2007)
Spatiotopic selectivity of BOLD responses to visual motion in human area MT. Nat Neurosci 10:249-255. CrossRef Medline

DeSouza JF, Dukelow SP, Vilis T (2002) Eye position signals modulate early dorsal and ventral visual areas. Cereb Cortex 12:991-997. CrossRef Medline

Deutschländer A, Marx E, Stephan T, Riedel E, Wiesmann M, Dieterich M, Brandt T (2005) Asymmetric modulation of human visual cortex activity during 10 degrees lateral gaze (fMRI study). Neuroimage 28:4-13. CrossRef Medline

DeYoe EA, Carman GJ, Bandettini P, Glickman S, Wieser J, Cox R, Miller D, Neitz J (1996) Mapping striate and extrastriate visual areas in human cerebral cortex. Proc Natl Acad Sci U S A 93:2382-2386. CrossRef Medline

Engbert R, Mergenthaler K (2006) Microsaccades are triggered by low retinal image slip. Proc Natl Acad Sci U S A 103:7192-7197. CrossRef Medline

Engel SA, Rumelhart DE, Wandell BA, Lee AT, Glover GH, Chichilnisky EJ, Shadlen MN (1994) fMRI of human visual cortex. Nature 369:525. CrossRef Medline

Engel SA, Glover GH, Wandell BA (1997) Retinotopic organization in human visual cortex and the spatial precision of functional MRI. Cereb Cortex 7:181-192. CrossRef Medline

Galletti C, Battaglini PP (1989) Gaze-dependent visual neurons in area V3A of monkey prestriate cortex. J Neurosci 9:1112-1125. Medline

Galletti C, Battaglini PP, Fattori P (1995) Eye position influence on the parieto-occipital area PO (V6) of the macaque monkey. Eur J Neurosci 7:2486-2501. CrossRef Medline

Gardner JL, Merriam EP, Movshon JA, Heeger DJ (2008) Maps of visual space in human occipital cortex are retinotopic, not spatiotopic. J Neurosci 28:3988-3999. CrossRef Medline

Groh JM, Trause AS, Underhill AM, Clark KR, Inati S (2001) Eye position influences auditory responses in primate inferior colliculus. Neuron 29: 509-518. CrossRef Medline

Hansen KA, Kay KN, Gallant JL (2007) Topographic organization in and near human visual area V4. J Neurosci 27:11896-11911. CrossRef Medline

Huk AC, Dougherty RF, Heeger DJ (2002) Retinotopy and functional subdivision of human areas MT and MST. J Neurosci 22:7195-7205. Medline

Jay MF, Sparks DL (1984) Auditory receptive fields in primate superior colliculus shift with changes in eye position. Nature 309:345-347. CrossRef Medline

Kay KN, Naselaris T, Prenger RJ, Gallant JL (2008) Identifying natural images from human brain activity. Nature 452:352-355. CrossRef Medline

Lal R, Friedlander MJ (1990) Effect of passive eye position changes on retinogeniculate transmission in the cat. J Neurophysiol 63:502-522. Medline

Larsson J, Heeger DJ (2006) Two retinotopic visual areas in human lateral occipital cortex. J Neurosci 26:13128-13142. CrossRef Medline

Lehky SR, Peng X, McAdams CJ, Sereno AB (2008) Spatial modulation of primate inferotemporal responses by eye position. PLoS ONE 3:e3492. CrossRef Medline

McKyton A, Zohary E (2007) Beyond retinotopic mapping: the spatial representation of objects in the human lateral occipital complex. Cereb Cortex 17:1164-1172. Medline

Morel A, Bullier J (1990) Anatomical segregation of two cortical visual pathways in the macaque monkey. Vis Neurosci 4:555-578. CrossRef Medline

Nestares O, Heeger DJ (2000) Robust multiresolution alignment of MRI brain volumes. Magn Reson Med 43:705-715. CrossRef Medline

Ogawa S, Lee TM, Kay AR, Tank DW (1990) Brain magnetic resonance imaging with contrast dependent on blood oxygenation. Proc Natl Acad Sci U S A 87:9868-9872. CrossRef Medline

Pereira F, Mitchell T, Botvinick M (2009) Machine learning classifiers and fMRI: a tutorial overview. Neuroimage 45:S199-S209. CrossRef Medline

Pertzov Y, Avidan G, Zohary E (2011) Multiple reference frames for saccadic planning in the human parietal cortex. J Neurosci 31:1059-1068. CrossRef Medline

Rosenbluth D, Allman JM (2002) The effect of gaze angle and fixation distance on the responses of neurons in V1, V2, and V4. Neuron 33:143-149. CrossRef Medline

Schluppeck D, Glimcher P, Heeger DJ (2005) Topographic organization for delayed saccades in human posterior parietal cortex. J Neurophysiol 94: 1372-1384. CrossRef Medline 
Sereno MI, Dale AM, Reppas JB, Kwong KK, Belliveau JW, Brady TJ, Rosen BR, Tootell RB (1995) Borders of multiple visual areas in humans revealed by functional magnetic resonance imaging. Science 268 : 889-893. CrossRef Medline

Sharma J, Dragoi V, Tenenbaum JB, Miller EK, Sur M (2003) V1 neurons signal acquisition of an internal representation of stimulus location. Science 300:1758-1763. CrossRef Medline

Soechting JF, Flanders M (1992) Moving in three-dimensional space: frames of reference, vectors, and coordinate systems. Annu Rev Neurosci 15:167-191. CrossRef Medline

Stricanne B, Andersen RA, Mazzoni P (1996) Eye-centered, head-centered, and intermediate coding of remembered sound locations in area LIP. J Neurophysiol 76:2071-2076. Medline

Tootell RB, Hadjikhani N (2001) Where is "dorsal V4" in human visual cortex? Retinotopic, topographic and functional evidence. Cereb Cortex 11:298-311. CrossRef Medline

Tootell RB, Reppas JB, Kwong KK, Malach R, Born RT, Brady TJ, Rosen BR, Belliveau JW (1995) Functional analysis of human MT and related visual cortical areas using magnetic resonance imaging. J Neurosci 15:32153230. Medline
Trotter Y, Celebrini S (1999) Gaze direction controls response gain in primary visual-cortex neurons. Nature 398:239-242. CrossRef Medline

Wandell BA, Brewer AA, Dougherty RF (2005) Visual field map clusters in human cortex. Philos Trans R Soc Lond B Biol Sci 360:693-707. CrossRef Medline

Wang X, Zhang M, Cohen IS, Goldberg ME (2007) The proprioceptive representation of eye position in monkey primary somatosensory cortex. Nat Neurosci 10:640-646. CrossRef Medline

Wetherill GB, Levitt H (1965) Sequential estimation of points on a psychometric function. Br J Math Stat Psychol 18:1-10. CrossRef Medline

Williams AL, Smith AT (2010) Representation of eye position in the human parietal cortex. J Neurophysiol 104:2169-2177. CrossRef Medline

Xu Y, Wang X, Peck C, Goldberg ME (2011) The time course of the tonic oculomotor proprioceptive signal in area $3 \mathrm{a}$ of somatosensory cortex. J Neurophysiol 106:71-77. CrossRef Medline

Zhang M, Wang X, Goldberg ME (2008) Monkey primary somatosensory cortex has a proprioceptive representation of eye position. Prog Brain Res 171:37-45. CrossRef Medline

Zipser D, Andersen RA (1988) A back-propagation programmed network that simulates response properties of a subset of posterior parietal neurons. Nature 331:679-684. CrossRef Medline 